\title{
RESEARCH
}

\section{Fluoroquinolones compared with $\beta$-lactam antibiotics for the treatment of acute bacterial sinusitis: a meta-analysis of randomized controlled trials}

\author{
Drosos E. Karageorgopoulos MD, Konstantina P. Giannopoulou MD, \\ Alexandros P. Grammatikos MD MSc, George Dimopoulos MD, Matthew E. Falagas MD DSc
}

$\infty$

See related article page 865

\section{ABSTRACT}

Background: The presumed superiority of newer fluoroquinolones for the treatment of acute bacterial sinusitis is based on laboratory data but has not yet been established on clinical grounds.

Methods: We performed a meta-analysis of randomized controlled trials comparing the effectiveness and safety of fluoroquinolones and $\beta$-lactams in acute bacterial sinusitis.

Results: We identified 8 randomized controlled trials investigating the newer "respiratory" fluoroquinolones moxifloxacin, levofloxacin and gatifloxacin. In the primary effectiveness analysis involving 2133 intention-to-treat patients from 5 randomized controlled trials, the extent of clinical cure and improvement did not differ between fluoroquinolones and $\beta$-lactams (odds ratio [OR] 1.09, 95\% confidence interval $[\mathrm{Cl}] 0.85^{-1.39}$ ) at the test-ofcure assessment, which varied from 10 to 31 days after the start of treatment. Fluoroquinolones were associated with an increased chance of clinical success among the clinically evaluable patients in all of the randomized controlled trials (OR 1.29, $95 \%$ $\mathrm{Cl} 1.03-1.63$ ) and in 4 blinded randomized controlled trials (OR $1.45,95 \% \mathrm{Cl} 1.05-2.00$ ). There was no statistically significant difference between fluoroquinolones and amoxicillin-clavulanate (OR 1.24, 95\% Cl 0.93-1.65). Eradication or presumed eradication of the pathogens isolated before treatment was more likely with fluoroquinolone treatment than with $\beta$-lactam treatment (OR 2.11, 95\% Cl 1.09-4.08). In the primary safety analysis, adverse events did not differ between treatments (OR 1.17, $95 \% \mathrm{Cl}$ o.86-1.59). However, more adverse events occurred with fluoroquinolone use than with $\beta$-lactam use in 2 blinded randomized controlled trials. The associations described here were generally consistent when we included 3 additional studies involving other fluoroquinolones (ciprofloxacin and sparfloxacin) in the analysis.

Interpretation: In the treatment of acute bacterial sinusitis, newer fluoroquinolones conferred no benefit over $\beta$-lactam antibiotics. The use of fluoroquinolones as first-line therapy cannot be endorsed.

Une version française de ce résumé est disponible à l'adresse www.cmaj.ca/cgi/content/full/I78/7/845/DCI

CMAJ 2008;178(7):845-54
A cute bacterial sinusitis (more accurately known as rhinosinusitis, given that the nasal mucosa is commonly involved ${ }^{1}$ ) is one of the most frequent health disorders; ${ }^{2}$ it has an adverse impact on patients' quality of life $^{3}$ and accounts for nearly 3 million ambulatory care visits in the United States annually ${ }^{4}$ and substantial health care costs. ${ }^{5}$

Acute bacterial sinusitis typically follows an episode of viral upper respiratory tract illness. ${ }^{2}$ The diagnosis of bacterial disease in routine clinical practice is usually based on the presence of a constellation of clinical manifestations. ${ }^{1}$ The bacterial pathogens most commonly involved are Streptococcus pneumoniae, Haemophilus influenzae and, to a lesser degree, Moraxella catarrhalis. ${ }^{6,7}$ Over the years, these pathogens have acquired various degrees of resistance to many traditional antibiotics. ${ }^{8}$

The benefit of older antibiotics over placebo in the treatment of acute bacterial sinusitis appears limited, mostly because of the high success rate achieved with placebo. ${ }^{9,10}$ However, newer, third- and fourth-generation fluoroquinolones possess excellent in vitro activity against the most common respiratory pathogens,${ }^{11}$ and for this reason these drugs are often designated as "respiratory." Based on analysis of the available laboratory data, current guidelines give the newer fluoroquinolones the highest ranking, in terms of expected clinical effectiveness, among the antimicrobials used to treat acute bacterial sinusitis (although admittedly the difference is marginal).

The presumed clinical advantage of the respiratory fluoroquinolones over other classes of antimicrobials has not been clearly demonstrated in comparative clinical trials or metaanalyses. ${ }^{9,10}$ We aimed to comprehensively reassess the role of fluoroquinolones in the treatment of acute bacterial sinusitis, in terms of effectiveness and safety, by performing a metaanalysis of relevant randomized controlled trials.

From the Alfa Institute of Biomedical Sciences (AIBS) (all authors), Athens, Greece; the Department of Medicine (Grammatikos), Gennimatas General Hospital, Thessaloniki, Greece; the Intensive Care Unit (Dimopoulos), Attikon University Hospital, Athens, Greece; and the Department of Medicine (Falagas), Tufts University School of Medicine, Boston, Mass. 


\section{Methods}

\section{Data sources}

We searched MEDLINE (from July I965 to March 2007) for potentially relevant randomized controlled trials comparing fluoroquinolone antibiotics and $\beta$-lactams in the treatment of acute bacterial sinusitis. We chose $\beta$-lactam antibiotics as the comparator treatment because they constitute a wellestablished treatment option for acute bacterial sinusitis, ${ }^{12}$ they are frequently used in clinical practice, ${ }^{13}$ they retain sufficient in vitro activity against common respiratory pathogens, ${ }^{8}$ and they are among the agents with the highest expected clinical effectiveness, as recommended in current treatment guidelines. ${ }^{7}$ The following search strategy was used: "quinolones AND (sinusitis OR rhinosinusitis OR sinus infection)." We subsequently performed searches in the Scopus database and in the Cochrane Central Register of Controlled Trials to retrieve additional articles that might qualify for inclusion in our meta-analysis. Finally, the bibliographies of retrieved articles were hand-searched for references relevant to the subject of our meta-analysis.

\section{Study selection criteria}

Studies eligible for inclusion were randomized controlled trials comparing fluoroquinolone antibiotics with $\beta$-lactam antibiotics, in terms of effectiveness or safety, for the treatment of acute bacterial sinusitis. Trials involving patient populations with different clinical conditions were included only if they reported separately the data for patients with acute bacterial sinusitis or if the percentage of patients with acute bacterial sinusitis was greater than $70 \%$. Trials in languages other than English, French, German, Italian or Greek were not included in this meta-analysis.

For trials to be considered for inclusion in the metaanalysis, the diagnosis of acute bacterial sinusitis for patient enrolment had to have been based primarily on clinical criteria, whether or not further supported by radiologic or microbiologic criteria. The clinical diagnostic criteria used in each trial had to be in accord with the following definition of acute bacterial sinusitis: a disorder lasting no more than 28 days and comprising a constellation of signs and symptoms such as nasal congestion, purulent discharge, postnasal drip, facial pain or pressure, alteration in the sense of smell, fever, headache, cough, dental pain and halitosis. ${ }^{1,14}$

\section{Data extraction}

Two reviewers (A.P.G. and K.P.G.) independently evaluated all retrieved articles, on the basis of title and abstract, for eligibility for inclusion in the meta-analysis. The full text of potentially relevant articles was reviewed in detail.

The articles selected for inclusion were indexed and tabulated on the basis of first author, year of the study and country where the study took place. Data referring to the characteristics of each trial were extracted, including type and funding of study; definition of study population; patient inclusion and exclusion criteria; mean age and sex distribution of the study groups; numbers of patients enrolled, randomly assigned to treatment groups and evaluated; type, dosage and duration of study treatments; and use of additional medications. Also recorded were data referring to the definition of outcomes used in each study, the methods for evaluating the various outcomes and the time of the evaluation, as well as the actual measures of effectiveness and safety of the study treatments. Any differences between the 2 reviewers in terms of data extracted were resolved by discussion among all of the authors.

\section{Outcomes of the meta-analysis}

The primary comparison in this meta-analysis involved respiratory fluoroquinolones (defined as fluoroquinolones with enhanced activity against $S$. pneumoniae, which are recommended for the treatment of acute bacterial sinusitis ${ }^{7}$ ) versus $\beta$-lactam antibiotics. A secondary comparison involved all fluoroquinolones versus $\beta$-lactam antibiotics.

The primary effectiveness outcome of this meta-analysis was clinical success, which combined cure (according to clinical criteria) and substantial improvement in symptoms related to acute bacterial sinusitis, in the intention-to-treat population, defined as patients who were randomly assigned to receive a study treatment. The time of determination of the primary effectiveness outcome in each trial was termed the test-of-cure time point. The primary safety outcome of this meta-analysis was defined as the total number of adverse events recorded in patients who were evaluated for this outcome in the included trials.

The secondary effectiveness outcomes of the metaanalysis were the clinical success in the clinically evaluable population assessed at the test-of-cure time point of each included trial, and within a timeframe of 2I days since the beginning of study treatments, and the bacteriologic success of the bacteriologically evaluable population, defined as eradication or presumed eradication of the pathogens isolated before treatment through direct puncture of the paranasal sinuses or endoscopic sampling methods. The clinically evaluable population consisted of patients fulfilling the criteria for clinical evaluation, and the bacteriologically evaluable population consisted of patients fulfilling the criteria for bacteriologic evaluation, according to the criteria used in each study.

The secondary safety outcomes were the number of severe adverse events, as reported by the study investigators, the number of withdrawals from studies because of adverse events, and the number of relapses, defined by the reappearance of symptoms related to acute bacterial sinusitis after initial resolution. In addition, the specific types of adverse events for each treatment group that were most commonly reported in the included trials were pooled.

\section{Subset and sensitivity analyses}

We performed a subset analysis to compare respiratory fluoroquinolones with amoxicillin-clavulanate, in terms of clinical success at the test-of-cure time point, in the clinically evaluable population. Sensitivity analyses assessed the clinical success in the clinically evaluable population at test-of-cure time point, as well as total adverse events, in studies with a doubleblind or an investigator-blinded design. 


\section{Quality assessment}

The randomized controlled trials included in the meta-analysis were assessed for methodologic quality by considering the following parameters: reporting of adequate randomization procedures, allocation concealment, masking of the intervention, reporting of study withdrawals, reporting of patient crossovers between treatment arms and reporting of violation of the inclusion criteria.

\section{Data analysis and statistical methods}

For all statistical analyses performed, we calculated pooled odds ratios (ORs) and 95\% confidence intervals (CIs) using the Mantel-Haenszel fixed-effects model ${ }^{15}$ or, in the case of substantial statistical heterogeneity among the results of different studies, the DerSimonian-Laird random-effects model. ${ }^{16} \mathrm{We}$ assessed statistical heterogeneity between trials using the $\chi^{2}$ statistic $^{17}$ or, if no heterogeneity was detected by this method, the $\mathrm{I}^{2}$ statistic. ${ }^{18}$ We assessed publication bias by means of the funnel plot method using Egger's test. ${ }^{19,20}$

\section{Results}

\section{Selected trials}

We initially screened IgI articles that were retrieved from the MEDLINE database after application of the search criteria. Of these, 9 randomized controlled trials fulfilled the criteria for inclusion in our meta-analysis. Two additional trials eligible for inclusion were detected after a secondary search on the Scopus database, but no additional trials were identified in the Cochrane Central Register of Controlled Trials. A total of II trials ${ }^{21-31}$ were included in the meta-analysis (Figure I). The 2 reviewers independently reached the same conclusions regarding studies to be included.

\section{Characteristics of the selected trials}

The characteristics of the selected trials, specifically the type of trial, inclusion criteria used, definition of the study population, mean age and sex distribution of patients, numbers of patients enrolled and randomly assigned to treatment, and details of the treatments compared, are presented in Appendix I (available online at www.cmaj.ca/cgi/content/full/178/7/845 /DC2).

Eight of the II trials compared respiratory fluoroquinolones (specifically moxifloxacin [4 trials], levofloxacin [3 trials] or gatifloxacin [I trial]) with $\beta$-lactams. The remaining 3 trials investigated other fluoroquinolones (specifically ciprofloxacin [ 2 trials] and sparfloxacin [I trial]). The $\beta$ lactam comparators used in these trials were amoxicillin combined with clavulanic acid ( 5 trials), cefuroxime axetil ( 5 trials) and cefdinir ( $\mathrm{I}$ trial). All but I of the randomized controlled trials included in the meta-analysis had 2 treatment arms; the exception compared short-course (5-day) gatifloxacin treatment with standard-duration (Io-day) gatifloxacin treatment and with amoxicillin-clavulanate treatment. ${ }^{24} \mathrm{We}$ used the data for the standard-duration gatifloxacin treatment arm in this meta-analysis. In addition to the treatments being compared in these studies, adjunctive medications for symptomatic relief, mostly decongestants, antihistamines and analgesics, were permitted in 8 of the trials. ${ }^{22,24-30}$ Two of these trials reported the percentage of patients using such medications: $76 \%$ in one study ${ }^{28}$ and $83.5 \%$ in the other. ${ }^{29}$

Of the II randomized controlled trials included in this metaanalysis, 5 were open-label trials, 5 had a double-blind design, and I had an investigator-blinded design (see Appendix I, available at www.cmaj.ca/cgi/content/full/178/7/845/DC2).

Adequate randomization procedures were reported in 6 of the II trials, ${ }^{22,23,24,28-30}$ allocation concealment was reported in 3 trials, ${ }^{22,24,31}$ masking of the intervention was reported in 5 trials, ${ }^{22,24,26,28,29}$ information on patient withdrawals from the trials was reported in 7 trials, ${ }^{22,25-30}$ crossovers of patients between treatment arms were reported in I trial, ${ }^{27}$ and violation of the inclusion criteria was reported in 4 trials. ${ }^{26,28,29,31}$

In Io of the II trials, the fluoroquinolone was the experimental treatment and the $\beta$-lactam antibiotic the comparator; in the other trial, the fluoroquinolone (levofloxacin) served as

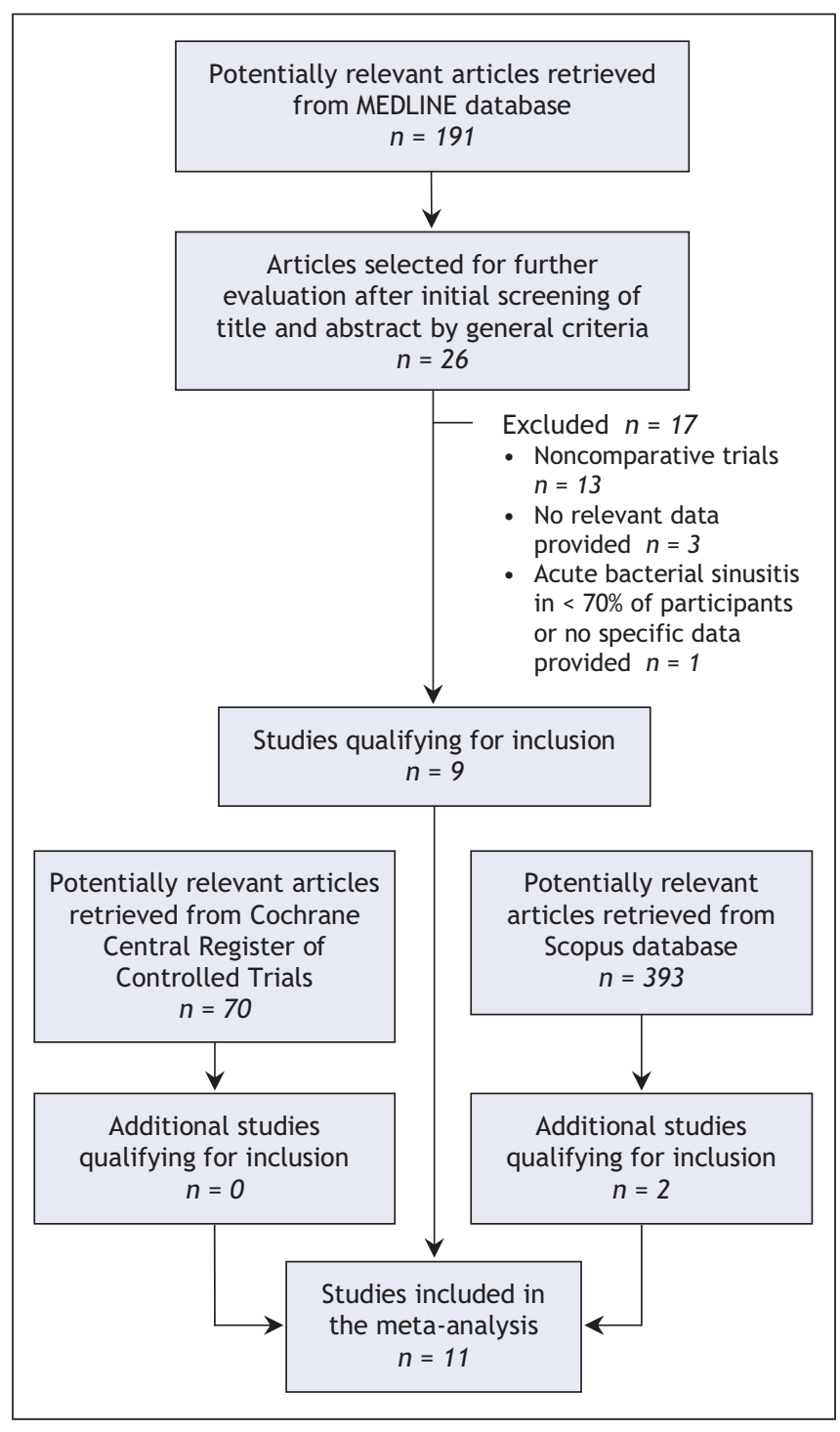

Figure 1: Flow diagram of the process for selecting articles for a meta-analysis of fluoroquinolones compared with $\beta$-lactam antibiotics in the treatment of acute bacterial sinusitis. 
the control treatment for an evaluation of the cephalosporin cefdinir. ${ }^{22}$ All but one of the included trials were designed to show statistical equivalence or noninferiority between the compared treatments; the exception ${ }^{23}$ was by far the smallest trial (in terms of number of patients enrolled) and therefore could not have been adequately powered to show superiority. Finally, for all of the trials except one, for which relevant data were not reported, ${ }^{31}$ either financial support was received from associated pharmaceutical companies or some of the authors were affiliated with the companies.

Among the patients included in the trials, the mean age was 4 I.I years, and $62.6 \%$ of the patients were women. All of the trials involved outpatients with presumed acute bacterial sinusitis; 2 of the trials also enrolled patients with acute exacerbation of chronic sinusitis, but these patients represented less than $30 \%$ of the whole patient group. ${ }^{23,29}$ In addition, 3 of

\section{Clinical success \\ Intention-to-treat population \\ Respiratory fluoroquinolones \\ Clinically evaluable population \\ Respiratory fluoroquinolones* \\ All fluoroquinolones* \\ Respiratory fluoroquinolones \\ v. amoxicillin-clavulanate \\ Blinded randomized controlled trials \\ Respiratory fluoroquinolones* \\ All fluoroquinolones* \\ Clinical success within 10-21 d \\ Respiratory fluoroquinolones* \\ All fluoroquinolones* \\ Bacteriologic success \\ Respiratory fluoroquinolones* \\ All fluoroquinolones*}

$2.11(1.09-4.08)$

$1.99(1.24-3.19)$

$1.29(1.03-1.63)$

$1.24(1.03-1.49)$

$1.24(0.93-1.65)$

$1.45(1.05-2.00)$

$1.36(1.04-1.77)$

$1.39(1.02-1.88)$

$1.32(1.03-1.71)$

0.2

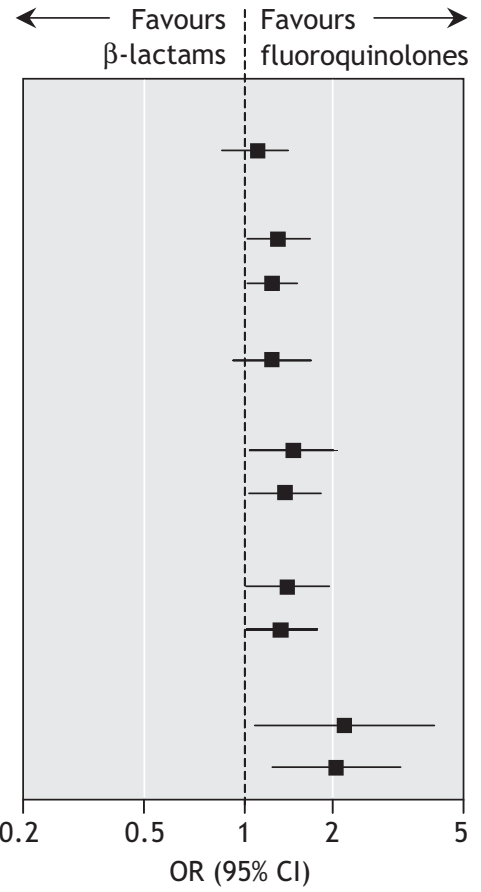

Relapse

Respiratory fluoroquinolones

Adverse events

Respiratory fluoroquinolones

All fluoroquinolones

Blinded randomized controlled trials

Respiratory fluoroquinolones*

All fluoroquinolones*

Severe adverse events

Respiratory fluoroquinolones*

All fluoroquinolones*

Withdrawals

Respiratory fluoroquinolones

All fluoroquinolones
$0.79(0.47-1.33)$

$1.17(0.86-1.59)$

$1.16(0.95-1.40)$

$1.54(1.19-2.00)$

$1.33(1.09-1.63)$

$0.53(0.30-0.95)$

$0.53(0.30-0.93)$

$1.35(0.94-1.95)$

$1.17(0.88-1.56)$

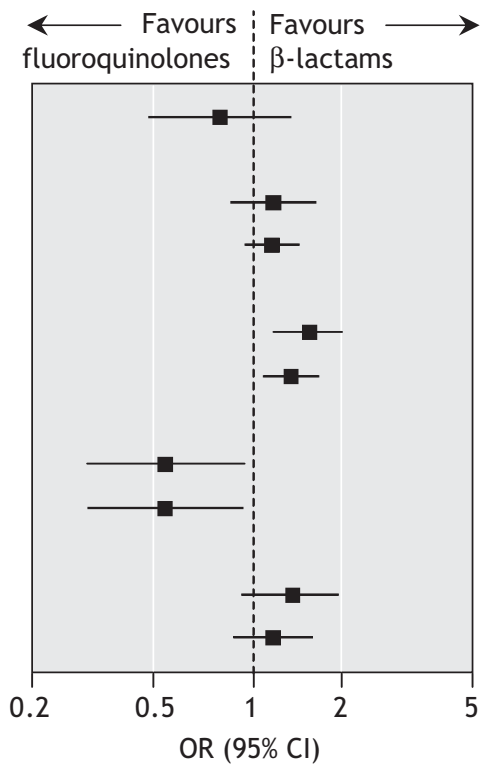

Figure 2: Pooled odds ratios (ORs) and $95 \%$ confidence intervals (Cls) of comparisons of effectiveness and safety between fluoroquinolones and $\beta$-lactam antibiotics. In both forest plots, an OR greater than 1 indicates an increased likelihood of the outcome with fluoroquinolone therapy. In the upper forest plot, an OR greater than 1 favours fluoroquinolone therapy; in the lower forest plot, an OR greater than 1 favours $\beta$-lactam therapy. An asterisk indicates a statistically significant difference. The vertical line represents no difference between compared treatments. See Methods for descriptions of each comparison. 
the included studies ${ }^{23,24,30}$ reported that a large number of the patients had a history of allergic rhinitis.

The diagnosis of acute bacterial sinusitis was based on various clinical criteria; radiologic criteria were also used in 8 of the trials (see Appendix I, available at www.cmaj.ca/cgi /content/full/I78/7/845/DC2).

\section{Time of determination of primary effectiveness outcome}

The time of determination of the primary effectiveness outcome (i.e., the time of the test-of-cure visit for the assessment of clinical success in each trial) varied from Io to $3 \mathrm{I}$ days after the beginning of study treatment (see Appendix 2, available at www.cmaj.ca/cgi/content/full/I78/7/845/DC2).

\section{Evaluation of outcomes}

Five of the studies, all involving respiratory fluoroquinolones, reported data on the clinical effectiveness of treatments in the intention-to-treat population, and all II studies reported relevant data regarding the clinically evaluable population. The criteria defining the clinically evaluable population consisted mainly of the requirement for adequate compliance with the assigned antimicrobial treatment, lack of use of additional antibiotics unless a clinical failure was noted and availability of the patient for the test-of-cure evaluation. By these criteria, $85.9 \%$ of the intention-to-treat population in the fluoroquinolone groups and $86.6 \%$ of the intention-to-treat population in the $\beta$-lactam groups were clinically evaluable.

The criteria used to define clinical cure or improvement, the primary outcome of this meta-analysis, differed among the studies (see Appendix 2, available at www.cmaj.ca/cgi/content/full $\left./ 178 / 7 / 845 / \mathrm{DC}_{2}\right)$. For example, in 6 of the II trials, the resolution of signs and symptoms of acute sinusitis had to be accompanied by resolution of the radiographic findings. ${ }^{21-23,27-29}$ Two of the studies provided no data on the combined outcome of cure or improvement but did report data on the complete resolution of signs and symptoms. ${ }^{21,31}$ Despite these differences, we entered all available relevant data in the analysis of the primary outcome.
In all of the 5 trials that assessed the bacteriologic efficacy of the treatments, ${ }^{21,23,26,29,3 \mathrm{I}}$ bacteriologic success for patients with no clinical manifestation of persistent infection corresponded mainly to presumed rather than microbiologically documented eradication of the pathogens that had been isolated before treatment. As for the methods used for bacteriologic sampling, specimens for culture were obtained exclusively by sinus puncture and aspiration in 2 trials; $;^{21,29}$ in the remaining 3 trials, sinus puncture was just one of the methods used, along with aspiration from the middle meatus or endoscopic sampling.

Specific data regarding adverse events were provided in all but 2 of the trials, the exceptions both involving comparison of respiratory fluoroquinolones with $\beta$-lactams. In these 2 trials, the incidence of drug-related adverse events was reported as generally comparable between the treatment groups..$^{22,24}$

\section{Comparisons of effectiveness}

Appendix 2 (available at www.cmaj.ca/cgi/content/full/178/7 1845/DC2) presents the detailed data that we considered for the analyses of effectiveness and safety of the compared treatments. Figure 2 presents the pooled ORs and 95\% CIs for all of the analyses performed. Detailed results for each of the analyses are presented in the following text.

\section{Clinical success}

All of the 5 studies that provided specific relevant data for the analysis of clinical success in the intention-to-treat population compared respiratory fluoroquinolones with $\beta$-lactam antibiotics. No statistically significant difference was observed between treatment groups at the test-of-cure time point (fixed-effects model for data from 5 randomized controlled trials with a total of $2 \mathrm{I} 33$ patients, OR 1.09 , 95\% CI o.85-I.39) (Figure 3).

Among the clinically evaluable patients, those who received respiratory fluoroquinolones (moxifloxacin, levofloxacin or gatifloxacin) had a greater chance of clinical success than those given $\beta$-lactams, as assessed at the test-of-cure time

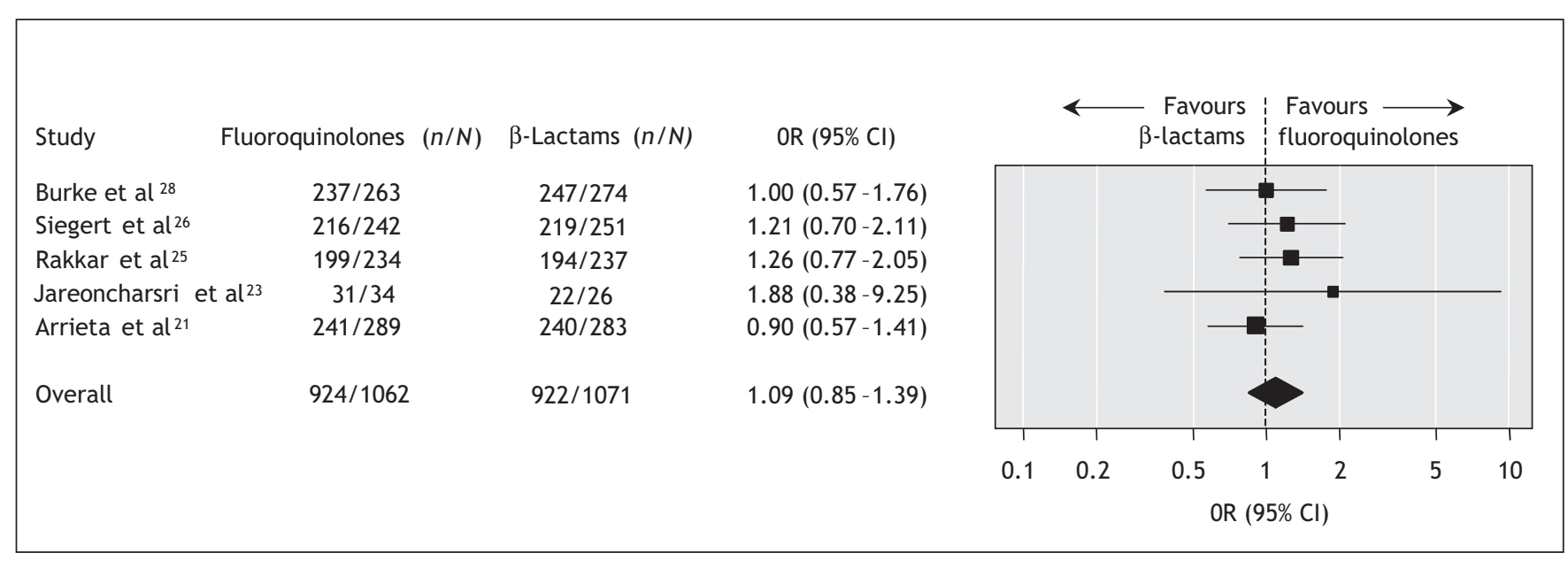

Figure 3: Meta-analysis of clinical success at the test-of-cure assessment of patients randomly assigned to treatment groups (intentionto-treat population) given either "respiratory" fluoroquinolones or $\beta$-lactam antibiotics. The vertical line represents no difference between compared treatments. 
point (fixed-effects model for 8 trials with a total of 2797 patients, OR I.29, 95\% CI I.03-I.63) (Figure 4). The same was true for patients who received any fluoroquinolone (including ciprofloxacin and sparfloxacin) relative to those given $\beta$ lactams (fixed-effects model for II trials with a total of 4640 patients, OR I.24, 95\% CI I.03-I.49) (Figure 4).

Clinically evaluable patients had a greater chance of clinical cure or improvement within 2I days after the start of study treatments if they received a respiratory fluoroquinolone rather than $\beta$-lactam treatment (fixed-effects model for 5 trials with a total of 1758 patients, OR I. $39,95 \%$ CI I.02-I.88). The same was true for patients who received any fluoroquinolone rather than $\beta$-lactam treatment (fixed-effects model for 7 trials with a total of 2382 patients, OR I. 32, 95\% CI I.03-I.7I).

In the sensitivity analysis, limited to the double-blinded or investigator-blinded randomized controlled trials, clinically evaluable patients had a greater chance of clinical success if they were given a fluoroquinolone rather than a $\beta$-lactam, both in the comparison of respiratory fluoroquinolones with $\beta$ lactams (fixed-effects model for 4 trials with a total of $I_{402}$ patients, OR I.45, 95\% CI I.05-2.00) and in the comparison of all fluoroquinolones with $\beta$-lactams (fixed-effects model for 6 trials with a total of 2026 patients, OR I.36, 95\% CI I.04-I.77).

We found no statistically significant difference in clinical success between clinically evaluable patients given a respiratory fluoroquinolone and those given amoxicillin-clavulanate (fixed-effects model for 5 trials with a total of $\mathrm{I} 663$ patients, OR I.24, 95\% CI 0.93-I.65).

\section{Bacteriologic success}

Eradication or presumed eradication of pathogens that were isolated before treatment was more likely if patients received a respiratory fluoroquinolone rather than a $\beta$-lactam (fixedeffects model for 3 trials with a total of 506 patients, OR 2.II, $95 \% \mathrm{CI}$ 1.09-4.08) or if they received any fluoroquinolone rather than a $\beta$-lactam (fixed-effects model for 5 trials with a total of 868 patients, OR I.99, 95\% CI I.24-3.19).

\section{Relapse}

Data for relapse of signs and symptoms of sinusitis were available only from trials that compared respiratory fluoroquinolones and $\beta$-lactams. We found no statistically significant difference in terms of relapse, as recorded by patients' follow-up visits (fixed-effects model for 4 trials with a total of I599 patients, OR 0.79, 95\% CI 0.47-I.33).

\section{Comparisons of safety}

\section{Adverse events}

The rate of adverse events did not differ significantly between patients given a respiratory fluoroquinolone and those who received a $\beta$-lactam (random-effects model for 6 trials with a total of 2732 patients, OR I.I7, 95\% CI o.86-I.59) (Figure 5). The same was true for patients given any fluoroquinolone relative to those given a $\beta$-lactam (random-effects model for 9 trials with a total of 5018 patients, OR I.I6, 95\% CI 0.95-I.40) (Figure 5). The specific types of adverse events reported for

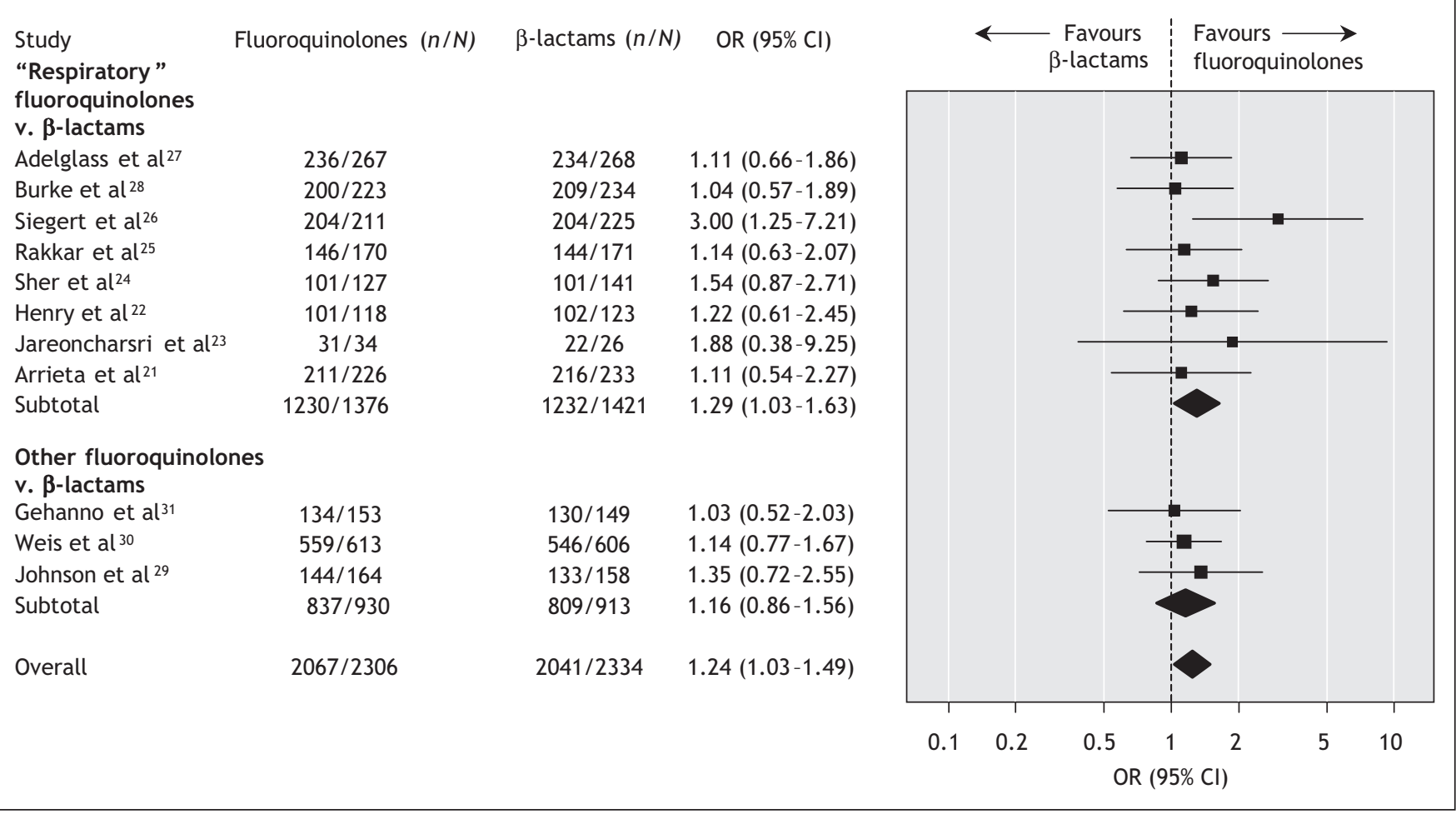

Figure 4: Meta-analysis of clinical success at the test-of-cure assessment of patients fulfilling the criteria for clinical evaluation (clinically evaluable population) given either "respiratory" or other fluoroquinolones or given $\beta$-lactam antibiotics. The vertical line represents no difference between compared treatments. 
patients treated with any fluoroquinolone and with $\beta$-lactams were total gastrointestinal adverse events ( 4 trials, 2828 patients, incremental rate $10.2 \%$ v. $9.8 \%$ ), diarrhea ( 8 trials, 4650 patients, $3.8 \%$ v. $7.2 \%$ ), nausea (7 trials, 4379 patients, $5.9 \%$ v. $3.1 \%$ ), adverse events affecting the body as a whole (4 trials, 3053 patients, $5.9 \%$ v. $5.5 \%$ ), abdominal pain ( 5 trials, 2672 patients, $2.7 \%$ v. $2.4 \%$ ), vaginitis ( 5 trials, I 990 female patients, I. $7 \%$ v. $4.6 \%$ ) and adverse events affecting the nervous system ( 3 trials, 2454 patients, $2.7 \%$ v. $0.9 \%$ ).

In blinded randomized controlled trials, significantly more adverse events were associated with the use of respiratory fluoroquinolones than with $\beta$-lactam antibiotics (fixedeffects model for 2 trials with a total of I030 patients, OR I.54, 95\% CI I.I9-2.00). The same was true for patients given any fluoroquinolone rather than a $\beta$-lactam antibiotic (fixedeffects model for 4 trials with a total of 1905 patients, OR I.33, 95\% CI I.09-I.63).

\section{Severe adverse events}

Significantly fewer adverse events were assessed as severe among patients who received respiratory fluoroquinolones than among those who received $\beta$-lactams (fixed-effects model for 6 trials with a total of 2503 patients, OR $0.53,95 \%$ CI $0.30-0.95$ ). The same was true for patients given any fluoroquinolone rather than a $\beta$-lactam antibiotic (fixed-effects model for 7 trials with a total of 3004 patients, OR $0.53,95 \%$ CI 0.30-0.93).

\section{Withdrawals due to adverse events}

The chances of withdrawal from the trials because of adverse events were not statistically significantly different between patients given a respiratory fluoroquinolone and those given a $\beta$-lactam antibiotic (fixed-effects model for 8 trials with a to- tal of 3298 patients, OR I.35, 95\% CI 0.94-I.95). The same was true for patients given any fluoroquinolone rather than a $\beta$-lactam (fixed-effects model for II trials with a total of 5584 patients, OR I.I7, 95\% CI 0.88-I.56).

\section{Interpretation}

The main finding of our meta-analysis is that the so-called respiratory fluoroquinolones did not prove superior to $\beta$ lactams for the treatment of acute bacterial sinusitis in terms of the primary effectiveness outcome (clinical success [clinical cure or improvement]) or the primary safety outcome (total adverse events). However, in some of the secondary analyses, treatment outcomes were better among patients who received a respiratory fluoroquinolone than among those given a $\beta$-lactam antibiotic, specifically in the comparison of clinically evaluable populations (patients fulfilling the criteria for clinical evaluation), in the sensitivity analysis limited to blinded randomized controlled trials, and in terms of bacteriologic success (eradication or presumed eradication of the pathogens isolated before treatment). Yet patients who received a respiratory fluoroquinolone did not have greater clinical success than those given amoxicillin-clavulanate, nor did they have fewer relapses than patients given a $\beta$-lactam antibiotic. Furthermore, in the sensitivity analysis limited to blinded randomized controlled trials, the fluoroquinolone group had more total adverse events than the $\beta$-lactam group. Still, among all the relevant studies included in our metaanalysis, treatment with fluoroquinolones resulted in fewer severe adverse events.

The aforementioned associations did not change substantially when we included randomized controlled trials compar-

\begin{tabular}{|c|c|c|c|c|c|c|c|c|c|}
\hline Study & Fluoroquinolones $(n / N)$ & $\beta$-Lactams $(n / N)$ & OR $(95 \% \mathrm{Cl})$ & & $\stackrel{\leftarrow}{\text { fluoro }}$ & $\begin{array}{l}\text { Favo } \\
\text { nolo }\end{array}$ & $\begin{array}{l}\text { Favours } \\
\beta \text {-lacta }\end{array}$ & & \\
\hline \multicolumn{4}{|c|}{ "Respiratory" fluoroquinolones } & & & & & & \\
\hline Adelglass et al 27 & $114 / 297$ & $146 / 302$ & $0.67(0.48-0.92)$ & & & & & & \\
\hline Burke et $\mathrm{al}^{28}$ & $96 / 263$ & $70 / 274$ & $1.68(1.16-2.42)$ & & & & $\longrightarrow$ & & \\
\hline Siegert et $\mathrm{al}^{26}$ & $105 / 242$ & $88 / 251$ & $1.42(0.99-2.04)$ & & & & $=$ & & \\
\hline Rakkar et al 25 & $136 / 234$ & $124 / 237$ & $1.26(0.88-1.82)$ & & & & & & \\
\hline Jareoncharsri et al23 & $3 / 34$ & $2 / 26$ & $1.16(0.18-7.51)$ & & & & & & \\
\hline Arrieta et $\mathrm{l}^{21}$ & $93 / 289$ & $84 / 283$ & $1.12(0.79-1.60)$ & & & & & & \\
\hline Subtotal & $547 / 1359$ & $514 / 1373$ & $1.17(0.86-1.59)$ & & & & & & \\
\hline \multicolumn{10}{|c|}{ Other fluoroquinolones } \\
\hline Gehanno et $\mathrm{al}^{31}$ & 19/190 & $15 / 184$ & $1.25(0.62-2.55)$ & & & & & & \\
\hline Weis et $\mathrm{al}^{30}$ & $136 / 711$ & $115 / 700$ & $1.20(0.92-1.58)$ & & & & & & \\
\hline Johnson et al 29 & $115 / 250$ & $113 / 251$ & $1.04(0.73-1.48)$ & & & & & & \\
\hline Subtotal & $270 / 1151$ & $243 / 1135$ & $1.15(0.93-1.41)$ & & & & & & \\
\hline \multirow[t]{3}{*}{ Overall } & $817 / 2510$ & $757 / 2508$ & $1.16(0.95-1.40)$ & & & & & & \\
\hline & & & & 0.1 & 0.2 & 0.5 & 2 & 5 & 10 \\
\hline & & & & \multicolumn{6}{|c|}{ OR $(95 \% \mathrm{Cl})$} \\
\hline
\end{tabular}

Figure 5: Meta-analysis of adverse events reported among patients given "respiratory" or other fluoroquinolones or given $\beta$-lactam antibiotics. The vertical line represents no difference between compared treatments. 
ing any fluoroquinolone with a $\beta$-lactam antibiotic in the analysis. However, ciprofloxacin and sparfloxacin, the fluoroquinolones used in these additional studies, are not regarded as being as potent against $S$. pneumoniae, in microbiologic terms, as the newer fluoroquinolones, ${ }^{32}$ and they are not currently recommended for the treatment of acute bacterial sinusitis. ${ }^{7}$ Nonetheless, both were approved by the US Food and Drug Administration for this indication, although sparfloxacin was withdrawn from the US market in 200I. Despite their inferior bacteriologic activity, our inclusion of ciprofloxacin and sparfloxacin in this meta-analysis may be meaningful, given that these 2 agents share certain pharmacokinetic properties with the newer respiratory fluoroquinolones, specifically with regard to drug penetration into the sinus mucosa. ${ }^{33-35}$ In addition, from a safety standpoint, most of the adverse events observed with fluoroquinolones are thought to be related to a class effect rather than an agentspecific effect. ${ }^{36}$

In the remainder of this discussion, we focus on the respiratory fluoroquinolones, although many of the issues addressed may also be relevant to the comparison of all fluoroquinolones with $\beta$-lactam antibiotics.

Although fluoroquinolones were comparable to $\beta$-lactam antibiotics in terms of the effectiveness outcomes of this meta-analysis when the intention-to-treat population was examined, analysis of the clinically evaluable population showed a marginal advantage in favour of fluoroquinolone treatment. This apparent disparity can be explained by the fact that the intention-to-treat analysis generally provides a more conservative estimate of treatment effect. Moreover, data for the outcome of clinical success in the intention-totreat population were available for only 5 of the II included randomized controlled trials. However, the value of the intention-to-treat analysis is that it respects the random assignment of patients to study treatments and precludes bias in the selection of patients for evaluation, which is particularly important in nonblinded trials. In addition, the nature of the intention-to-treat analysis relates more closely to therapeutic decision-making in clinical practice.

In addition to the overall rate of resolution of symptoms in cases of acute bacterial sinusitis, the rapidity of symptom resolution is also important, because this condition adversely affects quality of life ${ }^{3}$ and is associated with substantial loss of productivity and associated sick leave expenditures in the workplace. ${ }^{37}$ We therefore performed a subanalysis assessing clinical effectiveness within 3 weeks after the start of treatment, which showed a marginal benefit of fluoroquinolones over $\beta$-lactams.

Some factors might blunt the demonstration of true differences between treatments in trials of acute bacterial sinusitis. The most important such factor is the expected enrolment of many patients with self-limiting viral disease, despite the use of clinical or radiologic diagnostic inclusion criteria. ${ }^{7,38-40}$ In fact, in this meta-analysis, the pooled percentage of patients with bacteriologically documented disease in the trials in which relevant studies were performed was $54 \%$. Additional factors that may confound the assessment of effectiveness of the studied treatments include the potential for sinusitis-like symptoms to persist despite bacteriologic cure $7,38,39$ and the likelihood of unbalanced use of symptom-relief medications among different treatment groups. ${ }^{41}$ In this regard, the greater bacteriologic success exhibited with respiratory fluoroquinolones relative to $\beta$-lactams may be important. Still, the analysis of clinical success may be the best indicator of routine clinical practice.

Among the 5779 patients enrolled in the trials included in this meta-analysis, no serious complications of sinusitis, such as orbital or intracranial spread, were reported. Although serious complications of sinusitis are rare among patients who receive appropriate treatment, ${ }^{13}$ the lack of such complications in the populations evaluated in this metaanalysis could be attributed to the fact that patients with a greater likelihood of a complicated course of disease (e.g., those with a history of chronic sinusitis or recurrent acute sinusitis) were excluded from a considerable number of the trials, ${ }^{21,25,26,28-30,34}$ even though they are frequently encountered in routine clinical practice. In addition, in all but one of the included studies, ${ }^{25}$ the duration of follow-up was limited to no more than I month after the end of therapy, which may have been inadequate for accurate estimation of the rates of recurrence and progression to chronic sinusitis.

From a safety standpoint, the primary analysis, which included all II studies, showed that the treatments were comparable in terms of total adverse events recorded. Most of the reported adverse events involved the gastrointestinal tract, with diarrhea being more frequent in the $\beta$-lactam group and nausea more frequent in the fluoroquinolones group. In the analysis of adverse events, significant statistical heterogeneity between trials was observed. This can be attributed to the findings of one trial, which had a lower rate of adverse events in the fluoroquinolone group, ${ }^{27}$ whereas the other trials had an opposing trend. When we omitted the one trial in a post hoc exploratory analysis, we found that adverse events were significantly more common among patients given a respiratory fluoroquinolone (fixed-effects model for 5 trials with a total of 2I33 patients, OR I.35, 95\% CI I.I3-I.62) or any fluoroquinolone (fixed-effects model for 8 trials with a total of 44I9 patients, OR I.26, 95\% CI I.IO-I.44) rather than a $\beta$ lactam antibiotic. In addition, in the sensitivity analysis limited to blinded randomized controlled trials, a higher rate of adverse events was associated with fluoroquinolone use than with $\beta$-lactam use. Notably, in a prior meta-analysis concerning treatment of skin and soft-tissue infections in $4352 \mathrm{pa}-$ tients, the use of fluoroquinolones was associated with significantly more adverse events than $\beta$-lactam use was. ${ }^{42}$

Nevertheless, adverse events characterized as severe were significantly less frequent among patients given a fluoroquinolone than among those given a $\beta$-lactam antibiotic. No cases of major arrhythmia, hepatitis, seizure or severe phototoxicity reaction, all of which are potentially serious adverse events associated with the use of some fluoroquinolones, ${ }^{36}$ were recorded among patients included in this meta-analysis. However, the absence of these adverse events could be partly attributed to the exclusion from clinical trials of patients who are prone to experience a serious adverse event associated with the study drugs. In fact, in 4 of the II trials included in 
this meta-analysis, patients were excluded if they had a history of seizure disorder, ${ }^{27}$ fluoroquinolone-related tendinopathy ${ }^{25,26,28}$ or a prolonged QRS interval. ${ }^{25,28}$ Moreover, some serious adverse events may be so rare that they will be identified only in large-scale postmarketing surveillance programs. This was the case for gatifloxacin, which has been withdrawn from the market because of its association with disturbances in glucose control. This drug was studied in only one of the trials included in our meta-analysis, and no adverse event of this type was reported among the 290 patients in the gatifloxacin group in that trial. ${ }^{24}$

Issues relating to bacterial drug resistance are also important in the choice of antimicrobial therapy. In fact, the major "respiratory" pathogens ( $S$. pneumoniae, $H$. influenzae and $M$. catarrhalis) have acquired resistance to multiple antibiotics, to various degrees. ${ }^{8}$ Of great concern is the increased prevalence of $S$. pneumoniae with reduced susceptibility to penicillin and, in parallel, increased resistance to other classes of antimicrobial agents. ${ }^{43}$ Although pneumococcal resistance to penicillin is relative and can be overcome with high-dose amoxicillin therapy in patients with sinusitis, ${ }^{44}$ the respiratory fluoroquinolones constitute a valuable therapeutic option. ${ }^{45}$ However, indiscriminate fluoroquinolone use may promote fluoroquinolone resistance in $S$. pneumoniae, which is often associated with multiple-drug resistance. ${ }^{46}$

Our meta-analysis had certain potential limitations. First, as in most meta-analyses of this kind, there was some variability in the characteristics of the included studies, relating chiefly to inclusion and evaluation criteria and the time of determination of the outcomes. Even so, for most of the analyses, the data retrieved did not exhibit significant heterogeneity. In addition, most of the included randomized controlled trials had some methodologic shortcomings. Moreover, the majority of the included trials, including all 5 of the nonblinded studies, were supported by pharmaceutical companies associated with the fluoroquinolone agents tested, a factor that may have generated bias in the assessment of outcomes. In this regard, the findings in the analysis of the blinded randomized controlled trials should be underscored.

Despite these limitations, the value of this meta-analysis lies in its combination of data from several randomized controlled trials, based on a non-inferiority design, which resulted in demonstration of differences in some aspects of the effectiveness and safety of the treatments. Most of the differences observed were of marginal statistical significance, and a randomized controlled trial of a very large sample would be required to manifest these differences. ${ }^{47}$

In conclusion, we found that respiratory fluoroquinolones were comparable to $\beta$-lactams for the treatment of acute bacterial sinusitis, in terms of the primary effectiveness outcome (clinical cure and improvement) and the primary safety outcome (total adverse events) examined. However, a propensity for more adverse events in association with fluoroquinolone use was observed in sensitivity and exploratory analyses. Thus, the use of respiratory fluoroquinolones as first-line therapy in the vast majority of patients with bacterial sinusitis, in whom the condition often has a benign clinical course, is not supported by the available evidence. The finding that presumed bacteriologic success was superior among fluoroquinolone-treated patients suggests that use of a respiratory fluoroquinolone may be considered in cases where $\beta$-lactam treatment has failed, particularly if the risk of infection with drug-resistant pathogens is a consideration.

This article has been peer reviewed.

Competing interests: None declared.

Contributors: Matthew Falagas conceived the study. Alexandros Grammatikos and Konstantina Giannopoulou performed the literature search, selected the studies and extracted the data. Drosos Karageorgopoulos and George Dimopoulos checked the accuracy of the extracted data. Drosos Karageorgopoulos performed the statistical analysis. All of the authors helped in interpreting the results of the analysis. Drosos Karageorgopoulos wrote the first draft of the manuscript; all of the authors contributed to revising the manuscript and approved the final version.

\section{REFERENCES}

I. Lanza DC, Kennedy DW. Adult rhinosinusitis defined. Otolaryngol Head Neck Surg I997; II7(3 Pt 2):SI-7.

2. Gwaltney JM Jr. Acute community-acquired sinusitis. Clin Infect Dis 1996;23:120923; quiz I224-5

3. Durr DG, Desrosiers MY, Dassa C. Impact of rhinosinusitis in health care delivery: the Quebec experience. J Otolaryngol 2001;30:93-7.

4. Schappert SM, Burt CW. Ambulatory care visits to physician offices, hospital outpatient departments, and emergency departments: United States, 2001-02. Vital Health Stat 132006 Feb;(I59):I-66.

5. Ray NF, Baraniuk JN, Thamer M, et al. Healthcare expenditures for sinusitis in I996: contributions of asthma, rhinitis, and other airway disorders. J Allergy Clin Immunol I999;I03:408-I4.

6. Gwaltney JM Jr, Scheld WM, Sande MA, et al. The microbial etiology and antimicrobial therapy of adults with acute community-acquired sinusitis: a fifteen-year experience at the University of Virginia and review of other selected studies. $J \mathrm{Al}$ lergy Clin Immunol I992;90:457-6I; discussion 462.

7. Anon JB, Jacobs MR, Poole MD, et al. Antimicrobial treatment guidelines for acute bacterial rhinosinusitis. Otolaryngol Head Neck Surg 2004;I30:I-45.

8. Jacobs MR, Felmingham D, Appelbaum PC, et al. The Alexander Project 1998-2000: susceptibility of pathogens isolated from community-acquired respiratory tract infection to commonly used antimicrobial agents. J Antimicrob Chemother 2003;52:229-46.

9. Williams JW Jr, Aguilar C, Cornell J, et al. Antibiotics for acute maxillary sinusitis [Cochrane review]. In: The Cochrane Library; Issue 3, 2003. Oxford: Update Software.

Io. Ip S, Fu L, Balk E, et al. Update on acute bacterial rhinosinusitis. Evid Rep Technol Assess (Summ) 2005 Jun;(124):I-3.

II. Shams WE, Evans ME. Guide to selection of fluoroquinolones in patients with lower respiratory tract infections. Drugs 2005;65:949-9I.

I2. Pakes GE, Graham JA, Rauch AM, et al. Cefuroxime axetil in the treatment of sinusitis. A review. Arch Fam Med 1994;3:165-75

13. Piccirillo JF, Mager DE, Frisse ME, et al. Impact of first-line vs second-line antibiotics for the treatment of acute uncomplicated sinusitis. JAMA 200I;286:1849-56.

I4. Benninger MS, Ferguson BJ, Hadley JA, et al. Adult chronic rhinosinusitis: definitions, diagnosis, epidemiology, and pathophysiology. Otolaryngol Head Neck Surg 2003;129(3 Suppl):SI-32.

I5. Mantel N, Haenszel W. Statistical aspects of the analysis of data from retrospective studies of disease. J Natl Cancer Inst 1959;22:719-48.

I6. DerSimonian R, Laird N. Meta-analysis in clinical trials. Control Clin Trials I986;7:177-88.

17. Cochran W. The combination of estimates from different experiments. Biometrics I954;IO:IOI-29.

I8. Higgins JP, Thompson SG, Deeks JJ, et al. Measuring inconsistency in metaanalyses. $B M J$ 2003;327:557-6o.

I9. Egger M, Davey Smith G, Schneider M, et al. Bias in meta-analysis detected by a simple, graphical test. BMJ I997;315:629-34.

20. Ioannidis JP, Trikalinos TA. The appropriateness of asymmetry tests for publication bias in meta-analyses: a large survey. CMAJ 2007;176:109I-6.

2I. Arrieta JR, Galgano AS, Sakano E, et al. Moxifloxacin vs amoxicillin/clavulanate in the treatment of acute sinusitis. Am J Otolaryngol 2007;28:78-82.

22. Henry DC, Kapral D, Busman TA, et al. Cefdinir versus levofloxacin in patients with acute rhinosinusitis of presumed bacterial etiology: a multicenter, randomized, double-blind study. Clin Ther 2004;26:2026-33.

23. Jareoncharsri P, Bunnag C, Fooanant $S$, et al. An open label, randomized comparative study of levofloxacin and amoxicillin/clavulanic acid in the treatment of purulent sinusitis in adult Thai patients. Rhinology 2004;42:23-9.

24. Sher LD, McAdoo MA, Bettis RB, et al. A multicenter, randomized, investigatorblinded study of 5 - and Io-day gatifloxacin versus ro-day amoxicillin/clavulanate in patients with acute bacterial sinusitis. Clin Ther 2002;24:269-8I. 
25. Rakkar S, Roberts K, Towe BF, et al. Moxifloxacin versus amoxicillin clavulanate in the treatment of acute maxillary sinusitis: a primary care experience. Int J Clin Pract 200I;55:309-I5.

26. Siegert R, Gehanno P, Nikolaidis P, et al. A comparison of the safety and efficacy of moxifloxacin (BAY 12-8039) and cefuroxime axetil in the treatment of acute bacterial sinusitis in adults. The Sinusitis Study Group. Respir Med 2000;94:337-44.

27. Adelglass J, DeAbate CA, McElvaine P, et al. Comparison of the effectiveness of levofloxacin and amoxicillin-clavulanate for the treatment of acute sinusitis in adults. Otolaryngol Head Neck Surg I999;120:320-7.

28. Burke T, Villanueva C, Mariano H Jr, et al. Comparison of moxifloxacin and cefuroxime axetil in the treatment of acute maxillary sinusitis. Sinusitis Infection Study Group. Clin Ther I999;21:1664-77.

29. Johnson PA, Rodriguez HP, Wazen JJ, et al. Ciprofloxacin versus cefuroxime axetil in the treatment of acute bacterial sinusitis. Sinusitis Infection Study Group. JOtolaryngol I999;28:3-I2.

30. Weis M, Hendrick K, Tillotson G, et al. Multicenter comparative trial of ciprofloxacin versus cefuroxime axetil in the treatment of acute rhinosinusitis in a primary care setting. Rhinosinusitis Investigation Group. Clin Ther 1998;20:921-32.

3I. Gehanno P, Berche P. Sparfloxacin versus cefuroxime axetil in the treatment of acute purulent sinusitis. Sinusitis Study Group. J Antimicrob Chemother 1996;37 (Suppl A):105-I4

32. George J, Morrissey I. The bactericidal activity of levofloxacin compared with ofloxacin, D-ofloxacin, ciprofloxacin, sparfloxacin and cefotaxime against Streptococcus pneumoniae. J Antimicrob Chemother 1997;39:719-23.

33. Wise R, Honeybourne $\mathrm{D}$. A review of the penetration of sparfloxacin into the lower respiratory tract and sinuses. JAntimicrob Chemother 1996;37(Suppl A):57-63.

34. Gehanno P, Darantiere S, Dubreuil C, et al. A prospective, multicentre study of moxifloxacin concentrations in the sinus mucosa tissue of patients undergoing elective surgery of the sinus. J Antimicrob Chemother 2002;49:82I-6.

35. Maier H, Zielinski D, Seigoleit A. Penetration of ciprofloxacin into the mucosa of the human maxillary sinus. JChemother 1989;I:570-I.

36. Ball P, Mandell L, Niki Y, et al. Comparative tolerability of the newer fluoroquinolone antibacterials. Drug Saf I999;2I:407-2I.

37. Goetzel RZ, Hawkins K, Ozminkowski RJ, et al. The health and productivity cost burden of the "top Io" physical and mental health conditions affecting six large U.S. employers in I999. J Occup Environ Med 2003;45:5-I4

38. Marchant CD, Carlin SA, Johnson CE, et al. Measuring the comparative efficacy of antibacterial agents for acute otitis media: the "Pollyanna phenomenon." J Pediatr 1992;120:72-7.

39. Poole MD. A mathematical therapeutic outcomes model for sinusitis. Otolaryngol Head Neck Surg 2004;130:46-50.

40. Scheid DC, Hamm RM. Acute bacterial rhinosinusitis in adults: part I. Evaluation. Am Fam Physician 2004;70:I685-92.

4I. Meltzer EO, Hamilos DL, Hadley JA, et al. Rhinosinusitis: establishing definitions for clinical research and patient care. Otolaryngol Head Neck Surg 2004;I3I:SI-62.

42. Falagas ME, Matthaiou DK, Vardakas KZ. Fluoroquinolones vs beta-lactams for empirical treatment of immunocompetent patients with skin and soft tissue infections: a meta-analysis of randomized controlled trials. Mayo Clin Proc 2006;8I: I553-66.

43. Doern GV, Heilmann KP, Huynh HK, et al. Antimicrobial resistance among clinical isolates of Streptococcus pneumoniae in the United States during 1999-2000, including a comparison of resistance rates since 1994-1995. Antimicrob Agents Chemother 200I;45:172I-9.

44. File TM Jr, Jacobs MR, Poole MD, et al. Outcome of treatment of respiratory tract infections due to Streptococcus pneumoniae, including drug-resistant strains, with pharmacokinetically enhanced amoxycillin/clavulanate. Int J Antimicrob Agents 2002;20:235-47.

45. Appelbaum PC. Resistance among Streptococcus pneumoniae: implications for drug selection. Clin Infect Dis 2002;34:1613-20.

46. Hooper DC. Fluoroquinolone resistance among gram-positive cocci. Lancet Infect Dis 2002;2:530-8.

47. Poole MD. Acute bacterial rhinosinusitis: clinical impact of resistance and susceptibility. Am J Med 2004;117(Suppl 3A):29S-38S

Correspondence to: Dr. Matthew E. Falagas, Alfa Institute of Biomedical Sciences (AIBS), 9 Neapoleos St., I5I 23 Marousi, Greece; fax $30210683-9605 ;$ m.falagas@aibs.gr 